\title{
BMJ Open Trajectories of CKD-MBD biochemical parameters over a 2-year period following diagnosis of secondary hyperparathyroidism: a pharmacoepidemiological study
}

Pierre Filipozzi, ${ }^{1,2}$ Carole Ayav, ${ }^{2,3}$ Willy Ngueyon Sime ${ }^{3}$ Emmanuelle Laurain, ${ }^{1,2}$ Michèle Kessler, ${ }^{1,2}$ Laurent Brunaud, ${ }^{4}$ Luc Frimat ${ }^{1,2,5}$

To cite: Filipozzi P, Ayav C, Ngueyon Sime W, et al. Trajectories of CKD-MBD biochemical parameters over a 2-year period following diagnosis of secondary hyperparathyroidism: a pharmacoepidemiological study. BMJ Open 2017;7: e011482. doi:10.1136/ bmjopen-2016-011482

- Prepublication history and additional material is available. To view please visit the journal (http://dx.doi.org/ 10.1136/bmjopen-2016011482).

Received 11 February 2016 Revised 20 June 2016 Accepted 31 August 2016

CrossMark

For numbered affiliations see end of article.

Correspondence to

Pr Luc Frimat;

I.frimat@chu-nancy.fr

\section{ABSTRACT}

Objectives: To define groups of patients according to the changes of biochemical parameters, that is, serum calcium, phosphate and parathyroid hormone (PTH), over a 2-year follow-up period using group-based multi-trajectory modeling (GBMM) among a cohort of dialysis patients with newly diagnosed secondary hyperparathyroidism (SHPT) (ie, PTH $\geq 500 \mathrm{ng} / \mathrm{L}$ for the first time) and to compare their patient characteristics and treatments.

Design: Pharmacoepidemiological study.

Setting: In the 12 dialysis units located in the French region of Lorraine.

Participants: A total of 269 dialysis patients with newly diagnosed SHPT were prospectively included from December 2009 to May 2012 and followed-up for 2 years.

Results: We identified four distinct trajectory groups: 'rapid PTH drop' experiencing a rapid and sharp decrease (over weeks) in PTH level associated with decreasing phosphate level within normal range $(n=34$; $12.7 \%$ ), 'gradual PTH decrease' experiencing a gradual and continuous decrease (over months) in PTH level and maintaining phosphate at a middle level throughout the study ( $n=98 ; 36.4 \%)$, 'slow PTH decrease with high phosphate' experiencing a slow decrease in PTH level associated with a relatively high phosphate level ( $n=105 ; 39.0 \%)$ and 'uncontrolled SHPT' with high levels of PTH and phosphate throughout the study $(n=32 ; 11.9 \%)$. Patients in the 'uncontrolled SHPT' group were significantly $(p<0.00001)$ younger than patients in other groups. Kidney Disease Improving Global Outcomes (KDIGO) targets for PTH, phosphate and calcium were reached simultaneously for $14.9 \%$ of patients at baseline and $16.7 \%$ at the end of the study. Patients were given cinacalcet more frequently at months 3 and 6 in the 'rapid PTH drop' and at month 24 in the 'uncontrolled SHPT' groups.

Conclusions: Over 2 years following a new SHPT diagnosis, a younger age and a higher rate of alkaline phosphatase were associated to a continuous uncontrolled SHPT. Patients with the lowest PTH at the

\section{Strengths and limitations of this study}

- The first study to apply group-based multitrajectory modeling (GBMM) in chronic kidney mineral and bone disorder (CKD-MBD).

- The EPHEYL study included all dialysis patients reporting newly diagnosed secondary hyperparathyroidism (SHPT) in a French administrative country, whereas most studies on biochemical assessment in SHPT patients included prevalent SHPT patients for whom data on disease duration were unavailable.

- The small sample size was counterbalanced by the rigorous methodology.

end of the follow-up tended to receive more often cinacalcet.

Trial registration number: ClinicalTrials.gov number, NCT02888639, post results.

\section{BACKGROUND AND OBJECTIVES}

Clinical practice guidelines on the management of chronic kidney mineral and bone disorder (CKD-MBD) recommend target levels for parathyroid hormone (PTH) in the range of two to nine times the upper normal limit and treatment modifications on the basis of trends rather than on a single value. ${ }^{1}$ Until now studies on CKD-MBD management assessed secondary hyperparathyroidism (SHPT) evolution using a single measurement value for a single biochemical parameter, mostly serum PTH level which is highly variable. Group-based multi-trajectory modeling (GBMM), in contrast, is a statistical approach recently used in clinical research for identifying distinct patterns of change within a population and estimating the likelihood for each individual of being assigned 
to a trajectory group. ${ }^{2}{ }^{3} \mathrm{~A}$ full and accurate description of illness trajectories may help clinicians plan and deliver appropriate care. To the best of our knowledge, trajectories of biochemical parameters of CKD-MBD in patients with newly diagnosed SHPT were never described with this statistical approach.

This study aimed to define groups of patients according to the changes of biochemical parameters, that is, serum calcium, phosphate and PTH over a 2-year follow-up period using GBMM among a cohort of dialysis patients with newly diagnosed SHPT, and to compare their patient characteristics and treatments.

\section{METHODS}

Settings

The pharmacoepidemiological EPHEYL (Étude PHarmacoÉpidémiologique de l'hYperparathyroïdie secondaire en Lorraine) study is a 2-year, open-cohort, prospective, observational study on incident SHPT, that is, newly diagnosed, with a 2-year follow-up, set in the 12 dialysis units located in the French region of Lorraine (public or private).

\section{Participants}

From 1 December 2009 to 31 May 2012, all patients who were on dialysis (haemodialysis or peritoneal dialysis) for at least 3 months were identified through the REIN registry-Region of Lorraine. ${ }^{4}$ Among them, those who experienced PTH $\geq 500 \mathrm{ng} / \mathrm{L}$ for the first time were included in our study. The PTH cut-off value of $500 \mathrm{ng} / \mathrm{L}$ was chosen at the time of 2003 K-DOQI. ${ }^{5}$ Indeed when we initiated the study, the updated Kidney Disease Improving Global Outcomes (KDIGO) recommendations were not effective and the advocated PTH target range was $150-300 \mathrm{ng} / \mathrm{L}$ in CKD stage 5 patients. ${ }^{5}$

All patients included in our study were prospectively followed-up for 2 years.

\section{Data collection}

Data collection was described in-depth elsewhere. ${ }^{6}{ }^{7}$ In brief, all sociodemographic and clinical data (biochemical parameters, outcomes, treatments) were retrieved from the REIN registry and prospectively collected from medical reports. ${ }^{4}$

A standardised form was used to collect data from medical records. A steering committee composed of an epidemiologist (CA) and a nephrologist (LF) reviewed all forms and medical records when collected biochemical data were out of international standards.

The following biochemical parameters were collected at the time of inclusion and during the study: PTH, calcaemia, phosphate, vitamin $\mathrm{D}$, alkaline phosphatase, albumin, haemoglobin and measured ionised calcium.

All drugs acting on phosphocalcic metabolism were collected during the study and classified into five groups: native vitamin $\mathrm{D}$, vitamin $\mathrm{D}$ receptor activators, calciumcontaining phosphate binder (CCPB), calcium-free phosphate binder (CFPB) and cinacalcet. A Charlson index was also calculated for each patient. ${ }^{8}$

\section{Statistical analysis}

Group-based multi-trajectory modeling

GBMM was used to identify distinct trajectories of phosphataemia, calcaemia and PTH. $^{9}{ }^{10}$ This statistical method is based on identifying groups of individuals following markedly distinct trajectories within a population and estimating probability for an individual to be assigned to a trajectory group. ${ }^{9}$ GBMM is a variant of group-based trajectory modeling devised to model trajectories for several parameters and to assess interrelationship of trajectories for these parameters without significantly increasing the total number of trajectory groups. $^{11} 12$

Serum phosphate and calcium levels as well as PTH/ assay upper limit ratio were modeled using the SAS (SAS Institute, http://www.sas.com) procedure, PROC TRAJ. ${ }^{911}$ This procedure is an extension of the statistical software SAS which fits a semiparametric mixture model to longitudinal data using the maximum likelihood method. The total number of measurements used was up to nine for each parameter (at inclusion and every 3 months over 2 years). The median was used when different values for a biochemical parameter had been collected for a patient in the same time interval. The Bayesian information criterion (BIC) was used to compare zero-inflated Poisson models with up to six trajectory groups. For each number of trajectories, we compared models defined by intercept with linear, quadratic or cubic models using the following formula: 2( $\triangle \mathrm{BIC})$ $>2 .{ }^{11}$ Each patient was assigned to a trajectory group for which the estimated probability of assignment was greatest. An assignment probability of 0.7 was considered a poor fit, while a value of 0.9 or higher was considered an excellent fit.

\section{Comparison of characteristics of the trajectory groups}

Qualitative variables were described by frequency and percentage and quantitative variables by mean and SD if the normality hypotheses (Shapiro-Wilks test) were fulfilled. Comparative bivariate analysis of all patient characteristics was performed using $\chi^{2}$ test or Fischer's exact test for qualitative variables, and analysis of variance or Kruskal-Wallis non-parametric test for Gaussian quantitative variables. Multivariate analyses of variance (MANOVA) were performed to compare the biochemical parameters of trajectory groups not used in the GBMM approach. When a difference was found, bivariate comparisons were performed using Bonferroni test or $\chi^{2}$ test by adjusting the $\alpha$ value using the Bonferroni correction. A comparative survival analysis between trajectory groups was also performed using the log-rank test.

\section{Model robustness}

We carried out a random sample among two-thirds of the patients of our population $(\mathrm{N}=179)$ to check the 
robustness of the model and account for uncertainties associated to the distribution of trajectory groups. These randomly selected patients were assigned to a trajectory group by iterating on the GBMM model. Then model calibration was performed using $\chi^{2}$ test and the Hosmer-Lemeshow test. Distribution of trajectory groups and assignment probabilities were assessed as recommended by Nagin and Odgers. ${ }^{10}$

\section{Sensitivity analysis}

An alternative model by iterating statistical analyses was devised by excluding patients for whom less than four biochemical values were collected at four time periods. We considered that these patients were at increased risk of being less likely to be assigned to a group.

We calculated the Cohen's kappa coefficient by excluding patients with less than four biochemical values or not in order to assess if distribution of trajectory groups was consistent.

\section{Missing data}

Latent trajectory model may be used if any biochemical parameter is missing. When the first day of a treatment was missing data and this treatment had not been actually reported in the quarterly report, we considered that this treatment began at mid-quarter, that is, 45 days before first reporting. Likewise, when the last day of a treatment was missing data, its discontinuation was considered to happen 45 days after the last reporting.

Statistical analyses were performed using SAS software, V.9.3 (SAS Institute, Cary, North Carolina, USA). A significance threshold of 0.05 was adopted for all analyses.

\section{RESULTS}

Among the 305 patients enrolled in the EPHEYL cohort, 269 patients with a PTH level $\geq 500 \mathrm{ng} / \mathrm{L}$ were included in this study. The mean duration for follow-up was 620.0 \pm 195.3 days. A total of 83 patients (30.9\%) left the study before the end with a length of follow-up of 371.2 \pm 184.5 days; among them, 54 died, 26 underwent kidney transplantation, 2 moved outside the study area and 1 had recovered from renal function and stopped dialysis treatment.

Several measurements for serum levels of PTH, phosphate and calcium were reported per patient: $6.9 \pm 2.2$ PTH values/patient (median of 8; 25th-75th centile: 6-9), 7.8 \pm 2.2 phosphate values/patient (median of 9; 25th-75th centile: 7-9) and 7.8 \pm 2.0 calcium values/ patient (median of $9 ; 25$ th-75th centile).

\section{Characteristics of four distinct trajectory groups}

The GBMM analysis using the zero-inflated Poisson modeling option allowed the identification of four distinct trajectories over a 2-year period after SHPT diagnosis (figure 1). The first group, identified as the 'rapid PTH drop' group, included $12.7 \%$ of patients $(\mathrm{N}=34)$ experiencing a rapid and sharp decrease in serum PTH level associated with decreasing serum phosphate level within normal range. Serum calcium levels were lower at the start of the study in this group when compared to other groups. The second group, identified as the 'gradual PTH decrease' group, included $36.4 \%$ of patients $(n=98)$ experiencing a gradual and continuous decrease in serum PTH level and maintaining serum phosphate at a middle level throughout the study. The third group, identified as the 'slow PTH decrease with high phosphate' group, included $39.0 \%$ of patients $(\mathrm{N}=105)$ experiencing a slow decrease in serum PTH level associated with a relatively high serum phosphate level. The fourth group, identified as the 'uncontrolled SHPT' group, included $11.9 \%$ of patients $(\mathrm{N}=32)$ with
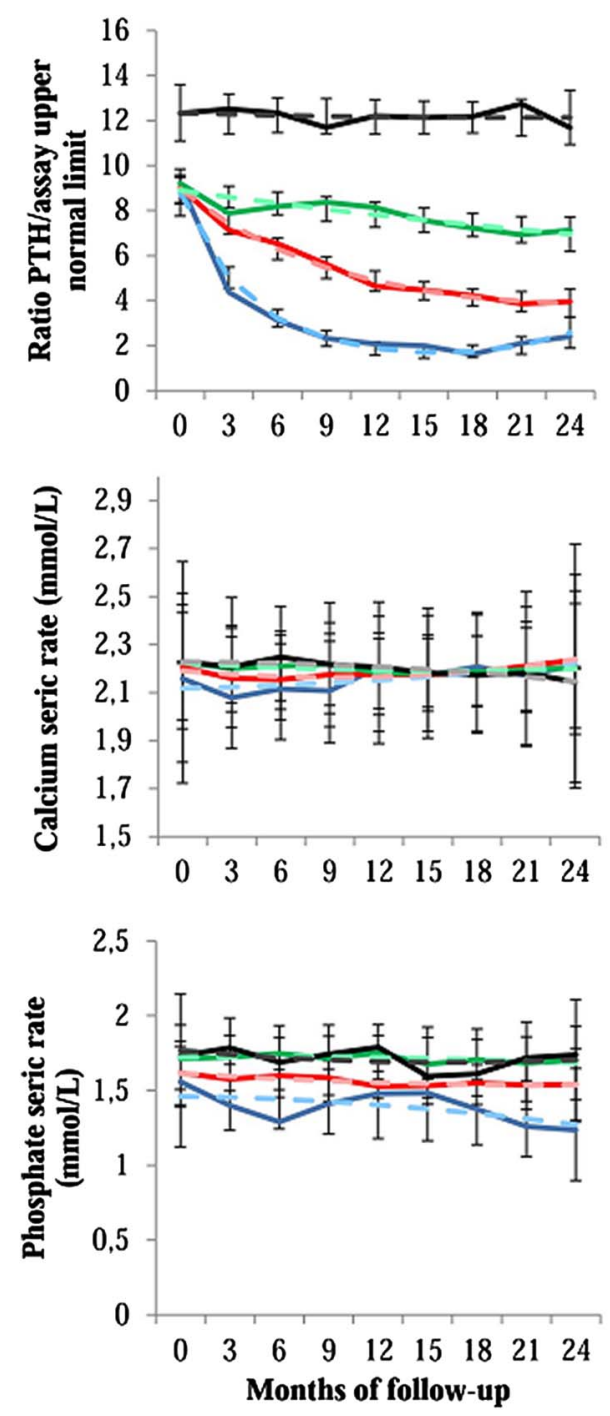

Figure 1 Trajectories for parathyroid hormone, calcium and phosphate over the two years after diagnosis of a severe secondary hyperparathyroidism. The solid lines indicate the observed trajectories, and the dashed lines indicate the predicted trajectories with 95\% Cls. Blue, 'rapid PTH drop' group; red: 'gradual PTH decrease' group; green, 'slow PTH decrease with high phosphate' group; black, 'uncontrolled SHPT' group. PTH, parathyroid hormone; SHPT, secondary hyperparathyroidism. 
high serum levels of PTH and phosphate throughout the study. Patients of this group had a significantly higher $\mathrm{PTH} /$ assay upper limit ratio at inclusion in the study (see online supplementary table S1).

Comparison of other biochemical values collected during the 24-month follow-up between groups is presented in table 1.

The group 'uncontrolled SHPT' had a significantly $(p<0.0001)$ higher rate of alkaline phosphatase when compared with other groups using MANOVA.
Table 2 highlights descriptive statistics of patient characteristics at inclusion by the trajectory group. There were no difference of survival between groups $(\mathrm{p}=0.24)$.

The three KDIGO targets were simultaneously reached for calcium, phosphate and PTH by $14.9 \%$ (40/ $269)$ and $16.7 \%(32 / 192)$ of patients at inclusion and at 24 months, respectively (see online supplementary table 2). There was no significant difference between groups (see online supplementary table 2 ). In the statistical analyses using the $\chi^{2}$ or Fischer's exact test, the

Table 1 Biochemical parameters during the follow-up according to trajectory groups

\begin{tabular}{|c|c|c|c|c|c|c|c|c|c|c|c|c|c|}
\hline & \multicolumn{3}{|c|}{$\begin{array}{l}\text { 'Rapid PTH drop' } \\
\text { group } \\
\mathrm{N}=34(12.7 \%)\end{array}$} & \multicolumn{3}{|c|}{$\begin{array}{l}\text { “Gradual PTH } \\
\text { decrease" group } \\
\mathrm{N}=98(36.4 \%)\end{array}$} & \multicolumn{3}{|c|}{$\begin{array}{l}\text { 'Slow PTH decrease } \\
\text { with high phosphate' } \\
\text { group } \\
\mathrm{N}=105(39.0 \%)\end{array}$} & \multicolumn{3}{|c|}{$\begin{array}{l}\text { 'Uncontrolled SHPT' } \\
\text { group } \\
\mathrm{N}=32(11.9 \%)\end{array}$} & \multirow[b]{2}{*}{ p Value } \\
\hline & $\mathbf{N}$ & Mean & SD & $\overline{\mathbf{N}}$ & Mean & SD & $\mathbf{N}$ & Mean & SD & $\overline{\mathbf{N}}$ & Mean & SD & \\
\hline \multicolumn{14}{|c|}{ Serum $25-\mathrm{OH}$ vitamin D level (ng/mL) } \\
\hline At baseline & 31 & 17.2 & 11.8 & 67 & 18.7 & 12.2 & 68 & 20.1 & 16.2 & 22 & 22.5 & 13.2 & 0.53 \\
\hline At 3 months & 18 & 27.2 & 17.2 & 30 & 20.4 & 12.0 & 36 & 23.4 & 15.1 & 14 & 24.5 & 13.4 & 0.45 \\
\hline At 6 months & 15 & 31.3 & 21.0 & 37 & 21.9 & 11.4 & 38 & 22.1 & 15.0 & 9 & 27.6 & 15.5 & 0.15 \\
\hline At 9 months & 15 & 30.0 & 14.4 & 34 & 22.7 & 15.2 & 33 & 24.5 & 15.3 & 13 & 21.4 & 8.1 & 0.36 \\
\hline At 12 months & 15 & 30.5 & 14.4 & 37 & 26.8 & 14.5 & 37 & 27.7 & 34.6 & 15 & 23.9 & 13.8 & 0.89 \\
\hline At 15 months & 15 & 23.0 & 11.6 & 34 & 24.4 & 13.5 & 26 & 28.1 & 15.9 & 10 & 17.7 & 8.7 & 0.22 \\
\hline At 18 months & 15 & 30.5 & 16.7 & 26 & 27.0 & 18.2 & 36 & 28.3 & 14.2 & 11 & 26.3 & 16.0 & 0.90 \\
\hline At 21 months & 11 & 32.8 & 11.1 & 33 & 27.1 & 16.6 & 29 & 28.2 & 14.8 & 15 & 21.1 & 10.6 & 0.23 \\
\hline At 24 months & 10 & 36.9 & 17.1 & 31 & 26.5 & 18.0 & 24 & 25.8 & 13.9 & 13 & 24.1 & 12.0 & 0.22 \\
\hline \multicolumn{14}{|c|}{ Serum alkaline phosphatase level (UI/L) } \\
\hline At baseline & 34 & 94.5 & 36.1 & 96 & 102.0 & 46.1 & 104 & 98.3 & 44.5 & 31 & 162.1 & 199.2 & $<0.001$ \\
\hline At 3 months & 31 & 101.1 & 48.6 & 85 & 98.0 & 41.7 & 96 & 101.3 & 48.0 & 28 & 176.8 & 185.4 & $<0.0001$ \\
\hline At 6 months & 30 & 89.1 & 35.4 & 82 & 97.6 & 36.0 & 97 & 103.1 & 48.8 & 26 & 184.5 & 149.0 & $<0.0001$ \\
\hline At 9 months & 29 & 79.4 & 32.0 & 76 & 97.9 & 41.0 & 94 & 100.4 & 40.3 & 25 & 200.2 & 169.1 & $<0.0001$ \\
\hline At 12 months & 27 & 78.1 & 27.6 & 76 & 96.9 & 50.1 & 86 & 102.9 & 43.1 & 29 & 168.3 & & $<0.0001$ \\
\hline At 15 months & 25 & 85.2 & 39.9 & 65 & 101.7 & 74.0 & 79 & 107.7 & 58.6 & 29 & 165.7 & 134.3 & 0.0001 \\
\hline At 18 months & 24 & 93.3 & 44.3 & 67 & 96.2 & 38.9 & 69 & 103.5 & 53.9 & 25 & 158.3 & 117.7 & $<0.001$ \\
\hline At 21 months & 21 & 82.0 & 33.8 & 62 & 94.9 & 36.4 & 68 & 103.3 & 48.2 & 25 & 151.1 & 81.3 & $<0.0001$ \\
\hline At 24 months & 20 & 86.8 & 47.1 & 60 & 92.7 & 35.0 & 58 & 105.9 & 49.7 & 22 & 184.5 & 182.0 & $<0.0001$ \\
\hline \multicolumn{14}{|c|}{ Serum albumin level (g/L) } \\
\hline At baseline & 33 & 35.4 & 5.6 & 92 & 36.4 & 4.3 & 102 & 35.8 & 4.5 & 31 & 37.4 & 4.4 & 0.28 \\
\hline At 3 months & 28 & 35.4 & 3.7 & 80 & 35.5 & 5.0 & 88 & 35.9 & 4.8 & 27 & 37.1 & 4.9 & 0.47 \\
\hline At 6 months & 31 & 35.8 & 5.0 & 74 & 35.6 & 5.3 & 88 & 36.9 & 4.8 & 25 & 37.2 & 5.4 & 0.28 \\
\hline At 9 months & 26 & 34.5 & 5.3 & 70 & 35.2 & 5.0 & 85 & 36.5 & 4.7 & 29 & 38.2 & 4.7 & 0.01 \\
\hline At 12 months & 26 & 33.8 & 5.5 & 68 & 34.9 & 5.4 & 77 & 35.4 & 5.0 & 25 & 37.4 & 5.4 & 0.10 \\
\hline At 15 months & 23 & 33.4 & 6.5 & 61 & 35.0 & 6.0 & 73 & 35.6 & 4.8 & 27 & 37.6 & 5.5 & 0.06 \\
\hline At 18 months & 24 & 32.9 & 6.9 & 61 & 35.4 & 5.7 & 70 & 35.8 & 5.7 & 25 & 36.5 & 5.5 & 0.14 \\
\hline At 21 months & 19 & 33.5 & 6.0 & 59 & 35.6 & 4.7 & 70 & 36.1 & 4.7 & 27 & 37.6 & 5.0 & 0.04 \\
\hline At 24 months & 17 & 35.1 & 3.6 & 54 & 35.6 & 5.1 & 65 & 36.2 & 5.4 & 21 & 38.1 & 3.3 & 0.18 \\
\hline \multicolumn{14}{|c|}{ Serum bicarbonate level (mmol/L) } \\
\hline At baseline & 34 & 22.4 & 3.3 & 98 & 22.2 & 3.6 & 103 & 22.1 & 3.3 & 32 & 22.1 & 4.3 & 0.97 \\
\hline At 3 months & 33 & 23.3 & 3.2 & 91 & 23.2 & 3.4 & 100 & 22.4 & 3.4 & 29 & 21.1 & 3.8 & 0.02 \\
\hline At 6 months & 32 & 23.1 & 2.6 & 87 & 23.4 & 3.7 & 97 & 22.6 & 3.9 & 30 & 22.1 & 2.7 & 0.26 \\
\hline At 9 months & 29 & 23.9 & 3.1 & 84 & 23.3 & 3.4 & 96 & 22.8 & 3.5 & 27 & 22.8 & 2.6 & 0.36 \\
\hline At 12 months & 27 & 23.7 & 3.1 & 80 & 22.8 & 3.1 & 88 & 23.1 & 4.0 & 25 & 22.0 & 3.2 & 0.31 \\
\hline At 15 months & 26 & 23.7 & 3.4 & 72 & 23.4 & 3.8 & 79 & 22.9 & 3.6 & 27 & 22.8 & 3.2 & 0.66 \\
\hline At 18 months & 26 & 24.2 & 3.1 & 72 & 23.3 & 3.7 & 80 & 23.1 & 4.0 & 27 & 22.0 & 3.1 & 0.16 \\
\hline At 21 months & 23 & 24.1 & 3.2 & 68 & 23.9 & 3.6 & 76 & 23.2 & 3.2 & 27 & 22.2 & 3.2 & 0.10 \\
\hline At 24 months & 21 & 24.1 & 2.6 & 63 & 24.3 & 3.8 & 73 & 23.5 & 3.5 & 26 & 22.6 & 3.0 & 0.15 \\
\hline
\end{tabular}

Bold indicates statistical significance, $p<0.05$.

*Analysis of variance or Wilcoxon's test used to compare continuous variables.

PTH, parathyroid hormone; SHPT, secondary hyperparathyroidism. 
'uncontrolled SHPT' group was less likely to reach KDIGO targets for PTH at inclusion and at 24 months than the 'gradual PTH decrease' group $(\mathrm{p}=0.003$ at inclusion; $\mathrm{p}=0.0002$ at 24 months) and the 'slow PTH decrease with high phosphate' group $(\mathrm{p}<0.0001$ at inclusion; $\mathrm{p}<0.0001$ at 24 months).

\section{Description of clinical practice}

The average weekly duration of dialysis treatment at the inclusion was 12.0 \pm 2.0 hours without any significant difference between the trajectory patterns.
A total of 157 of patients $(58.4 \%)$ received at least one prescription of cinacalcet throughout the follow-up. At the end of the follow-up, 38 patients of these 157 (24.2\%) versus 9 of the 108 patients who never received cinacalcet had a PTH value under two times the upper normal limit of the assay $(\mathrm{p}<0.001)$.

Vitamin D analogues, native vitamin D, CFPB and CCPB were prescribed at least once throughout the follow-up in $85(31.6 \%), 210(78.4 \%), 178(66.2 \%)$ and $213(79.2 \%)$ patients, respectively. The total length of the prescription period for each therapeutic class was not statistically different between trajectory groups (table 3 ).

Table 2 Baseline characteristics of patients according to trajectory groups

\begin{tabular}{|c|c|c|c|c|c|c|c|c|c|c|c|c|c|}
\hline & \multicolumn{3}{|c|}{$\begin{array}{l}\text { 'Rapid PTH drop' } \\
\text { group } \\
\mathrm{N}=34(12.7 \%)\end{array}$} & \multicolumn{3}{|c|}{$\begin{array}{l}\text { ‘Gradual PTH } \\
\text { decrease’ group } \\
\mathrm{N}=98(36.4 \%)\end{array}$} & \multicolumn{3}{|c|}{$\begin{array}{l}\text { 'Slow PTH decrease } \\
\text { with high phosphate' } \\
\text { group } \\
\mathrm{N}=105(39.0 \%)\end{array}$} & \multicolumn{3}{|c|}{$\begin{array}{l}\text { 'Uncontrolled } \\
\text { SHPT' group } \\
\mathrm{N}=32(11.9 \%)\end{array}$} & \multirow[b]{3}{*}{ p Value* } \\
\hline & & $\% /$ & & & $\% /$ & & & $\% /$ & & & $\% /$ & & \\
\hline & $\mathbf{N}$ & Mean & SD & $\mathbf{N}$ & Mean & SD & $\mathbf{N}$ & Mean & SD & $\mathbf{N}$ & Mean & SD & \\
\hline \multicolumn{14}{|l|}{ Sociodemographic data } \\
\hline Age at baseline & 34 & 68.8 & 14.6 & 98 & 68.5 & 13.6 & 105 & 67.2 & 14.9 & 32 & 54.1 & 19.4 & $<0.0001$ \\
\hline Gender male & 22 & 64.7 & & 59 & 60.2 & & 56 & 53.3 & & 20 & 62.5 & & 0.57 \\
\hline BMI & 34 & 28.3 & 6.9 & 98 & 28.0 & 7.2 & 105 & 27.4 & 6.3 & 32 & 26.5 & 6.3 & 0.66 \\
\hline $\mathrm{BMl}>30 \mathrm{~kg} / \mathrm{m}^{2}$ & 14 & 41.2 & & 33 & 33.7 & & 33 & 31.4 & & 6 & 18.8 & & 0.26 \\
\hline $\begin{array}{l}\text { Former or active tobacco } \\
\text { consumption }\end{array}$ & 8 & 23.5 & & 26 & 26.5 & & 33 & 31.4 & & 10 & 31.3 & & 0.76 \\
\hline Professional status & & & & & & & & & & & & & 0.004 \\
\hline Active & 2 & 6.5 & & 10 & 11.8 & & 16 & 15.8 & & 6 & 20.7 & & \\
\hline Retired & 23 & 74.2 & & 61 & 71.8 & & 66 & 65.3 & & 9 & 31.0 & & \\
\hline Other inactive & 6 & 19.4 & & 14 & 16.5 & & 19 & 18.8 & & 14 & 48.3 & & \\
\hline \multicolumn{14}{|l|}{ Medical data } \\
\hline $\begin{array}{l}\text { Comorbidities } \\
\text { potentially related to SHPT }\end{array}$ & 18 & 52.9 & & 59 & 60.2 & & 59 & 56.2 & & 12 & 37.5 & & 0.16 \\
\hline History of fracture & 5 & 14.7 & & 22 & 22.4 & & 24 & 22.9 & & 2 & 6.3 & & 0.14 \\
\hline \multicolumn{14}{|l|}{ Other comorbidities } \\
\hline Diabetes & 6 & 17.6 & & 20 & 20.4 & & 31 & 29.5 & & 9 & 28.1 & & 0.33 \\
\hline High blood pressure & 15 & 44.1 & & 40 & 40.8 & & 50 & 47.6 & & 19 & 59.4 & & 0.32 \\
\hline Existence of a disability & 5 & 14.7 & & 11 & 11.2 & & 14 & 13.3 & & 3 & 9.4 & & 0.89 \\
\hline Cardiovascular disease(s) & 18 & 52.9 & & 53 & 54.1 & & 52 & 49.5 & & 13 & 40.6 & & 0.60 \\
\hline Charlson comorbidity index & 34 & 5.7 & 2.2 & 98 & 5.9 & 2.1 & 105 & 5.6 & 1.9 & 32 & 4.4 & 2.3 & 0.005 \\
\hline History of kidney transplant & 2 & 5.9 & & 6 & 6.1 & & 9 & 8.6 & & 3 & 9.4 & & 0.88 \\
\hline Dialysis data & & & & & & & & & & & & & 0.52 \\
\hline \multicolumn{14}{|l|}{ Technique of dialysis } \\
\hline $\begin{array}{l}\text { Haemodialysis or } \\
\text { hemodiafiltration }\end{array}$ & 33 & 97.1 & & 90 & 91.8 & & 93 & 88.6 & & 29 & 90.6 & & \\
\hline Peritoneal dialysis & 1 & 2.9 & & 8 & 8.2 & & 12 & 11.4 & & 3 & 9.4 & & \\
\hline Dialysis vintage (months) & 34 & 41.2 & 73.2 & 98 & 46.3 & 67.8 & 105 & 39.7 & 68.0 & 32 & 50.8 & 67.5 & 0.45 \\
\hline Dialysate calcium & & & & & & & & & & & & & 0.77 \\
\hline \multicolumn{14}{|l|}{ concentration } \\
\hline $1.25 \mathrm{mmol} / \mathrm{L}$ & 3 & 8.8 & & 7 & 7.7 & & 14 & 13.6 & & 2 & 6.5 & & \\
\hline $1.50 \mathrm{mmol} / \mathrm{L}$ & 25 & 73.5 & & 69 & 75.8 & & 71 & 68.9 & & 24 & 77.4 & & \\
\hline $1.60 \mathrm{mmol} / \mathrm{L}$ & 1 & 2.9 & & 1 & 1.1 & & 0 & 0.0 & & 0 & 0.0 & & \\
\hline $1.75 \mathrm{mmol} / \mathrm{L}$ & 5 & 14.7 & & 14 & 15.4 & & 18 & 17.5 & & 5 & 16.1 & & \\
\hline Use of ESA & 27 & 81.8 & & 74 & 75.5 & & 73 & 69.5 & & 20 & 64.5 & & 0.34 \\
\hline
\end{tabular}


A total of 157 patients $(58.4 \%)$ received at least one dose of cinacalcet. Among them, 59 patients (21.9\%) had to stop taking cinacalcet treatment at least once. Regarding therapy with cinacalcet, the 'rapid PTH drop' group was most often treated at the inclusion and at 3 and 6 months whereas the 'uncontrolled SHPT' group was most often treated at 24 months (figure 2).

Biological hypocalcaemia developed in $79.6 \%$ of cinacalcet-treated patients and nausea occurred in $12.1 \%$ of them. All other adverse events occurred in $<1.0 \%$ of them. Regarding adverse events of cinacalcet, no significant difference was found between trajectory groups.

A total of eight patients $(3.0 \%)$ underwent a parathyroidectomy: four patients assigned in the 'uncontrolled SHPT' group after $464.5 \pm 175.3$ days of follow-up; two patients in the 'slow PTH decrease with high phosphate' group after a 368-day and 447-day follow-up (407.5 \pm 55.86 days); one patient in the 'gradual PTH decrease' group after a 181-day follow-up and one patient in the 'rapid PTH drop' after a 202-day follow-up.

\section{Validity of data}

Probability assignments were $0.89 \pm 0.17$ for the 'rapid PTH drop' group, $0.84 \pm 0.18$ for the 'gradual PTH decrease' group, $0.84 \pm 0.19$ for the 'slow PTH decrease with high phosphate' group and $0.99 \pm 0.23$ for the 'uncontrolled SHPT' group. The percentage of patients with a probability assignment to a group under 0.7 was $17.6 \%$ for the 'rapid PTH drop' group, $22.4 \%$ for the 'gradual PTH decrease' group, $27.6 \%$ for the 'slow PTH decrease with high phosphate' group and $6.3 \%$ for the 'uncontrolled SHPT' group. Model calibration was satisfactory for all trajectory groups as the Hosmer-Lemeshow test was not significant.
In figure 1 , there were no significant differences between observed values and those predicted by the statistical model irrespective of the length of follow-up and of the groups, except for PTH level in 'rapid PTH drop' and 'gradual PTH decrease' groups at 3 months.

After excluding 18 patients with less than four biochemical measurements throughout the follow-up, results remained significantly unchanged in the GBMM analysis (see online supplementary table S3). The distribution of patients into groups was preserved and the shape of trajectories was similar: $12.7 \%$ of patients were assigned in the 'rapid PTH drop' group, $35.5 \%$ in the 'gradual PTH decrease' group, $39.4 \%$ in the 'slow PTH decrease with high phosphate' group and $12.4 \%$ in the 'uncontrolled SHPT' group. The concordance between groups before and after excluding those patients was with a kappa coefficient of 0.99 .

\section{DISCUSSION}

In the EPHEYL study, we previously demonstrated: first, the benefit-risk balance of cinacalcet was presumably unfavourable in patients with a low PTH level; ${ }^{6}$ second, cinacalcet prescription was not associated with a better evolution of health-related quality of life after 1 year of treatment; ${ }^{7}$ third, cinacalcet was used in two-thirds of the patients, a weak proportion of them reaching KDIGO targets for PTH levels when compared to patients not treated with calcimimetics. ${ }^{13}$ Here we identify four distinct trajectories that mainly differed on the changes in serum PTH and phosphate levels over the first 2 years after diagnosis for SHPT in dialysis patients. These distinct progressive profiles actually reflect routine clinical situations. ${ }^{14}$ Patients in the 'rapid PTH drop' group experienced a drop of serum PTH level at the beginning of the study, that is, shortly after SHPT diagnosis, and were more likely to be overtreated

Table 3 Total length of treatments for SHPT according to trajectory groups

\begin{tabular}{|c|c|c|c|c|c|c|c|c|c|}
\hline \multirow[b]{2}{*}{ Length of treatments for SHPT } & \multicolumn{2}{|c|}{$\begin{array}{l}\text { 'Rapid PTH } \\
\text { drop' group } \\
\mathrm{N}=34(12.7 \%)\end{array}$} & \multicolumn{2}{|c|}{$\begin{array}{l}\text { 'Gradual PTH } \\
\text { drop' group } \\
\mathrm{N}=98(36.4 \%)\end{array}$} & \multicolumn{2}{|c|}{$\begin{array}{l}\text { 'Slow PTH } \\
\text { decrease with } \\
\text { high phosphate' } \\
\text { group } \\
\mathrm{N}=105(39.0 \%)\end{array}$} & \multicolumn{2}{|c|}{$\begin{array}{l}\text { 'Uncontrolled } \\
\text { SHPT' group } \\
\text { N=32 (11.9\%) }\end{array}$} & \multirow[b]{2}{*}{ p Value } \\
\hline & $\mathbf{N}$ & Mean (SD) & $\mathbf{N}$ & Mean (SD) & $\overline{\mathbf{N}}$ & Mean (SD) & $\mathbf{N}$ & Mean (SD) & \\
\hline \multicolumn{10}{|c|}{ Mean duration on the whole population (day/patient) } \\
\hline Vitamin D receptor activators & 34 & $96.9(198.6)$ & 98 & $116.3(223.7)$ & 105 & $123.3(213.1)$ & 32 & $143.0(226.0)$ & 0.85 \\
\hline Native vitamin D supplementation & 34 & $323.0(283.4)$ & 98 & $272.4(253.1)$ & 105 & $311.8(252.3)$ & 32 & $344.0(264.5)$ & 0.47 \\
\hline Cinacalcet & 34 & $268.2(256.0)$ & 98 & $219.8(257.0)$ & 105 & $198.5(241.5)$ & 32 & $276.4(246.3)$ & 0.31 \\
\hline Calcium-free phosphate binders & 34 & $249.4(280.9)$ & 98 & $301.3(293.7)$ & 105 & $317.9(289.2)$ & 32 & $410.5(295.8)$ & 0.15 \\
\hline Calcium-containing phosphate binders & 34 & $468.1(242.6)$ & 98 & $343.0(277.8)$ & 105 & $371.4(288.1)$ & 32 & $446.8(291.9)$ & 0.07 \\
\hline \multicolumn{10}{|c|}{ Mean duration in patients receiving the treatment (day/patient) } \\
\hline Vitamin D receptor activators & 9 & $365.9(227.8)$ & 28 & $406.9(239.0)$ & 38 & $340.6(227.3)$ & 14 & $326.8(239.1)$ & 0.64 \\
\hline Native vitamin D supplementation & 26 & $422.3(249.7)$ & 76 & $351.2(234.1)$ & 85 & $385.2(224.1)$ & 27 & $407.7(237.6)$ & 0.49 \\
\hline Cinacalcet & 22 & $414.4(198.7)$ & 58 & $371.3(234.7)$ & 63 & 330.9 (230.9) & 27 & $327.6(234.4)$ & 0.42 \\
\hline Calcium-free phosphate binders & 19 & $446.3(227.8)$ & 63 & $468.7(235.0)$ & 73 & $457.2(237.1)$ & 25 & $525.4(223.9)$ & 0.61 \\
\hline Calcium-containing phosphate binders & 31 & $513.4(201.8)$ & 73 & $460.4(221.6)$ & 85 & $458.8(249.3)$ & 26 & $549.8(216.2)$ & 0.23 \\
\hline
\end{tabular}




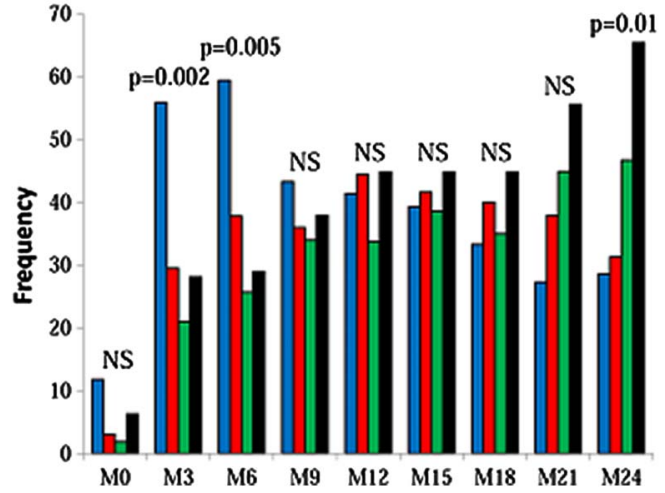

Figure 2 Cinacalcet prescription according to trajectory groups. Blue, 'rapid PTH drop' group; red: 'gradual PTH decrease' group; green, 'slow PTH decrease with high phosphate' group; black, 'uncontrolled SHPT' group. PTH, parathyroid hormone; SHPT, secondary hyperparathyroidism.

because the $\mathrm{PTH} /$ assay upper limit ratio was close to two from 9-month treatment to the end of the follow-up. Patients in the 'gradual PTH decrease' group and those in the 'slow PTH decrease with high phosphate' group experienced a more moderate decrease of PTH. Phosphataemia of the latter group, however, was more poorly controlled. Finally, the patients in the 'uncontrolled SHPT' group did not experience major changes in serum PTH and phosphate levels; such a clinical condition could account for a resistance pattern. In addition, the significant high level of serum alkaline phosphatase throughout the follow-up in the "uncontrolled SHPT' group highlights a more severe SHPT. ${ }^{15}$ Calcaemia was not found as a discriminatory variable in GBMM. This variable seemed less likely to vary widely within our population, whatever the group, as corroborated by a low SD for calcaemia in our follow-up. ${ }^{16}$ This could be explained by the fact that calcium level is a closely regulated physiological variable. ${ }^{17}$

Regarding baseline characteristics, differences between the 'uncontrolled SHPT' group and the other groups were observed and mainly concerned age, Charlson comorbidity index, professional status and current structure for dialysis unit. Age difference is likely to explain all differences regarding professional status and Charlson comorbidity index. Our results are consistent with those recently published by Parfrey et al. ${ }^{18}$ They showed that the more pronounced effect of cinacalcet in older patients than in younger patients may be explained by the association of bone demineralisation and vascular calcification with older age. In addition, serum alkaline phosphatase levels suggest that few patients immediately treated with cinacalcet had a high bone turnover. The difference for survival did not differ between groups, but this study did not aim to compare survival and was underpowered to assess any difference.

Some explanations may be suggested for the reasons underlying heterogeneous biological progression among these patients. The association between serum levels of
PTH and age is well-known and has been previously described in the literature. ${ }^{19}$ Nutritional status is also a well-known risk factor for low PTH. ${ }^{20}$ In our study, albumin levels did not differ in MANOVA but there was a trend for high rates in the 'uncontrolled SHPT' group with statistically significant differences at 9 and 21 months of follow-up.

Even if there was no statistically significant difference for total lengths of drug prescriptions, there were differences between groups for times of prescription. Patients of the 'rapid PTH drop' group were more likely to receive $\mathrm{CCPB}$ and cinacalcet at the beginning of the study and their PTH dropped simultaneously to a level close to two times the upper normal limit of the assay as recommended by the KDIGO guidelines. ${ }^{5}$ These data suggest that patients from this group were overtreated..$^{20}$ Patient profiles or trajectories observed in real-life are different from those identified in the first efficacy studies of cinacalcet, reflecting heterogeneity of patients in real-life whereas profiles of patients included in clinical trials are more homogenous due to a careful selection. In the EVOLVE trial, patients were rather young with a median age of 55 years, whereas patients in our study had a mean age of 66.3 years and a median age of 69 years, which was closer to the median age of the population in dialysis. ${ }^{21}$ The median serum calcium level was $2.175 \mathrm{mmol} / \mathrm{L}$ in our study, whereas it was $2.45 \mathrm{mmol} / \mathrm{L}$ in the EVOLVE trial. In addition, the EVOLVE population had a selected population profile excluding all patients with hypocalcaemia (serum calcium $<8.4 \mathrm{mg} / \mathrm{dL}$ ). Thus, pharmacoepidemiology studies are complementary to randomised controlled trials and provide a broad spectrum of information from real-life data.

Clinicians adhere to the $\mathrm{K} / \mathrm{DOQI}$ guidelines, and their main objective of prescribing is to reach advocated targets. In the EPHEYL study, frequencies of reaching simultaneously the three biochemical targets as recommended by the 2003 KDIGO guidelines were found to be low throughout the follow-up of CKD-MBD patients. Our results supported previous findings regarding the difficulty of reaching targets, particularly for phosphate. ${ }^{22}$ The proportion of deaths that occurred among the EPHEYL cohort by the end of the follow-up was consistent with the mortality rate of $\sim 120$ deaths for 1000 people/year obtained for dialysis patients in the age group (60-70) through the national REIN registry in $2012 .{ }^{23}$ In addition, our study showed $3.0 \%$ of patients undergoing parathyroidectomy, a low percentage compared to $6.6 \%$ and $7.1 \%$ of patients found in the COSMOS and Photo-Graph cohorts, respectively. ${ }^{22} 24$ This difference with previous cohorts should be seen in the light of the proportion of patients who received cinacalcet that accounted for $58.4 \%$. Comments should be pointed out regarding the most frequent use of cinacalcet instead of parathyroidectomy. First, we found a rather high frequency of adverse events in patients receiving cinacalcet. A higher frequency $(>90 \%$ of patients) was 
found in the EVOLVE randomised clinical trial, but reported adverse events were mainly related to digestive disorders whereas ours were mainly hypocalcaemia. ${ }^{21}$ Second, in most studies evaluating the efficacy of cinacalcet, parathyroidectomy was therefore reported as a treatment failure or an unwanted event. ${ }^{4526}$ No superiority of cinacalcet over parathyroidectomy has not been demonstrated yet, as well as no efficacy of cinacalcet on mortality from cardiovascular disease and all causes. ${ }^{24} 25$ In a medicoeconomic study, parathyroidectomy was more cost-effective than cinacalcet, except in patients with contraindication for surgery. ${ }^{27}$ Finally, previous findings demonstrated efficacy of parathyroidectomy on cardiovascular mortality. ${ }^{28}$ Lowest and highest values of PTH levels are probably associated with adynamic or hyperparathyroid bone disease, respectively. However, the majority of PTH levels will match with a grey zone where biochemical and analytical variability will hamper in-depth evaluation of CKD-MBD consequences. ${ }^{29}$

The major strength of this investigation lies in the use of GBMM which is a valuable and useful statistical tool for identifying groups of patients and for analysing large amounts of longitudinal data. This method avoids using subjective and objective criteria to identify groups. Another advantage of this method was to combine numerous biochemical measurements in our 2-year study whereas only two measurements at baseline and 12 months of follow-up were assessed in the study conducted by Block et al..$^{30}$ As highlighted by Stevens et al and advocated in the KDIGO guidelines, a single value for a phosphocalcic parameter should no more be considered, and a tool assessing biological trends in markers of mineral-bone disorders should be preferred. ${ }^{31} 32$ GBMM meets therefore both objectives. To the best of our knowledge, this is the first study in which GBMM was applied in CKD-MBD. Another strength was the exhaustive and consecutive data collection among all dialysis patients reporting a SHPT after dialysis initiation. The EPHEYL study included therefore incident SHPT patients who were more homogeneous than prevalent patients regarding onset of the disease and history of a shortened treatment regimen. Most studies on biochemical assessment in SHPT patients, in contrast, included prevalent SHPT patients for whom data on disease duration were unavailable. ${ }^{19} 2633$ The authors acknowledge several limitations. A number of nausea related to cinacalcet was likely not to be reported due to data collection on medical history and adverse events of cinacalcet from medical records. In addition, $500 \mathrm{ng} / \mathrm{L}$ was chosen as the cut-off PTH value before the publication of KDIGO clinical guidelines. It is now recognised that this value should not be interpreted as indicative of a confirmed diagnosis for SHPT. ${ }^{34}$ In our cohort, patients were not likely to have adynamic bone disease given mean values of PTH levels.

In conclusion, our study highlighted four groups of patients with different trajectories for phosphocalcic parameters using a modern and original statistical methodology. There were significant differences in age and alkaline phosphatase between groups. GBMM is a relevant and appropriate methodology for longitudinal data analysis among SHPT patients. Further studies are necessary on larger pools of patients using other biochemical parameters or combining GBMM with propensity score.

\section{Author affiliations}

${ }^{1}$ Department of Nephrology, University Hospital, Vandœuvre-lès-Nancy, France ${ }^{2}$ Néphrolor Network of Care, Vandœuvre-lès-Nancy, France

${ }^{3}$ Pôle S2R, Epidemiology and Clinical Evaluation, University Hospital, Vandœuvre-lès-Nancy, France

${ }^{4}$ Department of Hepatobiliary, Digestive and Endocrine Surgery, University Hospital, Vandœuvre-lès-Nancy, France

${ }^{5}$ Lorraine University, Paris Descartes University, Nancy, France

Acknowledgements The authors are extremely grateful to the patients who contributed to this study. They thank all nephrologists and hospital directors who participated in the study, and particularly the study nurse, Catherine Campagnac, and Brigitte Bourdillat for the manuscript editing.

Contributors PF, CA, LB and LF conceived the idea of the study. PF, CA, EL, MK, LB and LF designed the study. CA, LB and LF were responsible for data collection. $C A$ and $L F$ reviewed all forms and medical records when collected biochemical data were out of international standards. PF, CA, WNS and LF were responsible for undertaking data analysis and contributed to the interpretation of the results. PF produced the tables and graphs. The first draft of the manuscript was prepared by PF and was then circulated repeatedly among all authors for critical revision. PF, CA, WNS, EL, MK, LB and LF gave their final approval regarding submission for publication. PF, CA, WNS, EL, MK, LB and LF read and approved the final manuscript.

Funding This work was supported by a public funding through a Hospital Clinical Research Program ('PHRC inter-regional 2009') of the French Health Ministry and 'Agence de la Biomedicine' (2008).

\section{Competing interests None declared.}

Ethics approval This study was conducted in compliance with French regulations concerning pharmacoepidemiological studies. ${ }^{35}$ Approvals from the French data protection agency (CNIL: no. 904163) and from the Advisory Committee on information processing research in the field of health located in the region of Lorraine (CCTIRS: no. 0428) were obtained through the national REIN registry. An information sheet was displayed in all dialysis units, and each patient was given an individual written information sheet at the initiation of dialysis.

Provenance and peer review Not commissioned; externally peer reviewed.

Data sharing statement No additional data are available.

Open Access This is an Open Access article distributed in accordance with the Creative Commons Attribution Non Commercial (CC BY-NC 4.0) license, which permits others to distribute, remix, adapt, build upon this work noncommercially, and license their derivative works on different terms, provided the original work is properly cited and the use is non-commercial. See: http:// creativecommons.org/licenses/by-nc/4.0/

\section{REFERENCES}

1. Kidney Disease: Improving Global Outcomes (KDIGO) CKD-MBD Work Group. KDIGO clinical practice guideline for the diagnosis, evaluation, prevention, and treatment of Chronic Kidney Disease-Mineral and Bone Disorder (CKD-MBD). Kidney Int 2009;113:S1-30.

2. O'Hare AM, Batten A, Burrows NR, et al. Trajectories of kidney function decline in the 2 years before initiation of long-term dialysis. Am J Kidney Dis 2012;59:513-22.

3. Allen NB, Siddique J, Wilkins JT, et al. Blood pressure trajectories in early adulthood and subclinical atherosclerosis in middle age. JAMA 2014;311:490-7. 
4. Network and epidemiologic information in nephrology: kidney report 2010[In-ternet]. [quoted 2017 Mar 07]. Available: https://www. agence-biomedecine.fr/IMG/pdf/2012_rapport_annuel_rein.pdf

5. Moe SM, Chertow GM, Coburn JW, et al. Achieving NKF-K/DOQI bone metabolism and disease treatment goals with cinacalcet $\mathrm{HCl}$. Kidney Int 2005;67:760-71.

6. Laurain E, Ayav C, Erpelding ML, et al. Targets for parathyroid hormone in secondary hyperparathyroidism: is a "one-size-fits-all" approach appropriate? A prospective incident cohort study. BMC Nephrol 2014;15:132-43.

7. Filipozzi $P$, Ayav C, Erpelding ML, et al. Influence on quality of life from an early cinacalcet prescription for secondary hyperparathyroidism in dialysis. Pharmacoepidemiol Drug Saf 2015;24:187-96.

8. Charlson ME, Pompei P, Ales KL, et al. A new method of classifying prognostic comorbidity in longitudinal studies: development and validation. J Chronic Dis 1987;40:373-83.

9. Jones BL, Nagin DS, Roeder K. A SAS procedure based on mixture models for estimating developmental trajectories. Sociol Methods Res 2001;29:374-93.

10. Nagin DS, Odgers CL. Group-based trajectory modeling in clinical research. Annu Rev Clin Psychol 2010;6:109-38.

11. Jones BL, Nagin DS. Advances in group-based trajectory modeling and an SAS procedure for estimating them. Soc Methods Res 2007;35:542-71.

12. Nagin DS, Odgers CL. Group-based trajectory modeling (nearly) two decades later. J Quant Criminol 2010;26:445-53.

13. Brunaud L, Ngueyon Sime W, Filipozzi P, et al. Minimal impact of calcimimetics on the management of hyperparathyroidism in chronic dialysis. Surgery 2016;159:183-92.

14. Jean G, Souberbielle JC, Lorriaux C, et al. Clinical and biological forms of secondary hyperparathyroidism in dialysis patients. Nephrol Ther 2012;8:35-40.

15. Ureña P, Hruby M, Ferreira A, et al. Plasma total versus bone alkaline phosphatase as markers of bone turnover in hemodialysis patients. J Am Soc Nephrol 1996;7:506-12.

16. Levitt $\mathrm{H}$, Smith KG, Rosner MH. Variability in calcium, phosphorus, and parathyroid hormone in patients on hemodialysis. Hemodial Int 2009;13:518-25.

17. Courbebaisse M, Souberbielle JC. Phosphocalcic metabolism: regulation and explorations. Nephrol Ther 2011;7:118-38.

18. Parfrey PS, Drüeke TB, Block GA, et al. Evaluation of Cinacalcet $\mathrm{HCl}$ Therapy to Lower Cardiovascular Events (EVOLVE) Trial investigators. The effects of cinacalcet in older and younger patients on hemodialysis: the Evaluation of Cinacalcet $\mathrm{HCl}$ Therapy to Lower Cardiovascular Events (EVOLVE) trial. Clin J Am Soc Nephrol 2015;10:791-9.

19. Floege J, Kim J, Ireland E, et al. Serum iPTH, calcium and phosphate, and the risk of mortality in a European haemodialysis population. Nephrol Dial Transplant 2011;26:1948-55.

20. Andress DL. Adynamic bone in patients with chronic kidney disease. Kidney Int 2008;73:1345-54.

21. Chertow GM, Block GA, Correa-Rotter R, et al. Effect of cinacalcet on cardiovascular disease in patients undergoing dialysis. $N$ Engl J Med 2012;367:2482-94.
22. Fernández-Martín JL, Carrero JJ, Benedik M, et al. COSMOS: the dialysis scenario of CKD-MBD in Europe. Nephrol Dial Transplant 2013;28:1922-35.

23. Agence de la Biomédecine. Registre français des traitements de suppléance de l'insuffisance rénale chronique. Réseau, Epidémiologie, Information, Néphrologie. Rapport annuel 2012 http://www.soc-nephrologie.org/PDF/enephro/registres/rapport_2012/ rapport_2012.pdf

24. Pelletier S, Roth H, Bouchet JL, et al. Mineral and bone disease pattern in elderly haemodialysis patients. Nephrol Dial Transplant 2010;25:3062-70.

25. Block GA, Martin KJ, de Francisco AL, et al. Cinacalcet for secondary hyperparathyroidism in patients receiving hemodialysis. N Engl J Med 2004;350:1516-25.

26. Ureña $\mathrm{P}$, Jacobson $\mathrm{SH}$, Zitt $\mathrm{E}$, et al. Cinacalcet and achievement of the NKF/K-DOQI recommended target values for bone and mineral metabolism in real-world clinical practice-the ECHO observational study. Nephrol Dial Transplant 2009;24: 2852-9.

27. Komaba H, Moriwaki K, Goto S, et al. Cost-effectiveness of cinacalcet hydrochloride for hemodialysis patients with severe secondary hyperparathyroidism in Japan. Am J Kidney Dis 2012;60:262-71.

28. Goldenstein PT, Elias RM, Pires de Freitas do Carmo L, et al. Parathyroidectomy improves survival in patients with severe hyperparathyroidism: a comparative study. PLoS One 2013;8: e68870.

29. Garrett G, Sardiwal S, Lamb EJ, et al. PTH-a particularly tricky hormone: why measure it at all in kidney patients? Clin J Am Soc Nephrol 2013;8:299-12.

30. Block G, Do TP, Collins AJ, et al. Co-trending of parathyroid hormone and phosphate in patients receiving hemodialysis. Clin Nephrol 2016;85:142-51.

31. Stevens LA, Djurdjev O, Cardew S, et al. Calcium, phosphate, and parathyroid hormone levels in combination and as a function of dialysis duration predict mortality: evidence for the complexity of the association between mineral metabolism and outcomes. J Am Soc Nephrol 2004;15:770-9.

32. Moe SM, Drueke TB. Kidney disease: Improving Global Outcomes (KDIGO) CKD-MBD Work Group. KDIGO clinical practice guideline for the diagnosis, evaluation, prevention, and treatment of chronic kidney disease-mineral and bone disorder (CKD-MBD). Kidney Int 2009;76:S1-130.

33. Block GA, Klassen PS, Lazarus JM, et al. Mineral metabolism, mortality, and morbidity in maintenance hemodialysis. J Am Soc Nephrol 2004;15:2208-18.

34. Barreto FC, Barreto DV, Moysés RM, et al. K/ DOQI-recommended intact PTH levels do not prevent low-turnover bone disease in hemodialysis patients. Kidney Int 2008;73:771-7.

35. Claudot F, Alla F, Fresson J, et al. Ethics and observational studies in medical research: various rules in a common framework. Int J Epidemiol 2009;38:1104-8. 